\title{
Ultra-Wideband Transceivers for Cochlear Implants
}

\author{
Thomas Buchegger \\ Linz Center of Competence in Mechatronics, Altenbergerstraße 69, 4040 Linz, Austria \\ Email: thomas.buchegger@lcm.at

\section{Gerald Oßberger} \\ Linz Center of Competence in Mechatronics, Altenbergerstraße 69, 4040 Linz, Austria \\ Email: gerald.ossberger@lcm.at
}

Alexander Reisenzahn

Institute for Microelectronic, Johannes Kepler University of Linz, Altenbergerstraße 69, 4040 Linz, Austria

Email: alexander.reisenzahn@jku.at

\section{Erwin Hochmair}

Institute for Applied Physics, Leopold Franzens University of Innsbruck, Technikerstraße 25, 6020 Innsbruck, Austria Email:erwin.hochmair@uibk.ac.at

\author{
Andreas Stelzer \\ Institute for Communications and Information Engineering, Johannes Kepler University of Linz, Altenbergerstraße 69, \\ 4040 Linz, Austria \\ Email: a.stelzer@icie.jku.at
}

\section{Andreas Springer}

Institute for Communications and Information Engineering, Johannes Kepler University of Linz, Altenbergerstraße 69, 4040 Linz, Austria

Email: a.springer@icie.jku.at

Received 29 April 2004; Revised 21 January 2005

Ultra-wideband (UWB) radio offers low power consumption, low power spectral density, high immunity against interference, and other benefits, not only for consumer electronics, but also for medical devices. A cochlear implant (CI) is an electronic hearing apparatus, requiring a wireless link through human tissue. In this paper we propose an UWB link for a data rate of $1.2 \mathrm{Mbps}$ and a propagation distance up to $500 \mathrm{~mm}$. Transmitters with step recovery diode and transistor pulse generators are proposed. Two types of antennas and their filter characteristics in the UWB spectrum will be discussed. An ultra-low-power back tunnel diode receiver prototype is described and compared with conventional detector receivers.

Keywords and phrases: broadband antenna, cochlear implant, detector receiver, step recovery diode, back tunnel diode, ultrawideband.

\section{INTRODUCTION}

A cochlear implant is an electronic hearing device intended to help severely profoundly deaf individuals who gain little or no benefit from hearing aids [1]. It consists of two main parts: an internal implanted part and an external part

This is an open access article distributed under the Creative Commons Attribution License, which permits unrestricted use, distribution, and reproduction in any medium, provided the original work is properly cited. known as the speech processor. Sounds are picked up by a microphone which feeds the speech processor that converts the sound into electrical signals. The transmission system transmits the electrical signals, transcutaneous or percutaneous, to the implanted electrodes. The electrode or an electrode array, inserted into the cochlea by a surgeon, stimulates the auditory nerves. The majority of the CI manufacturers (Nucleus, Clarion, Med-El) use a transcutaneous link with an external and an implanted coil for the radio frequency link. 


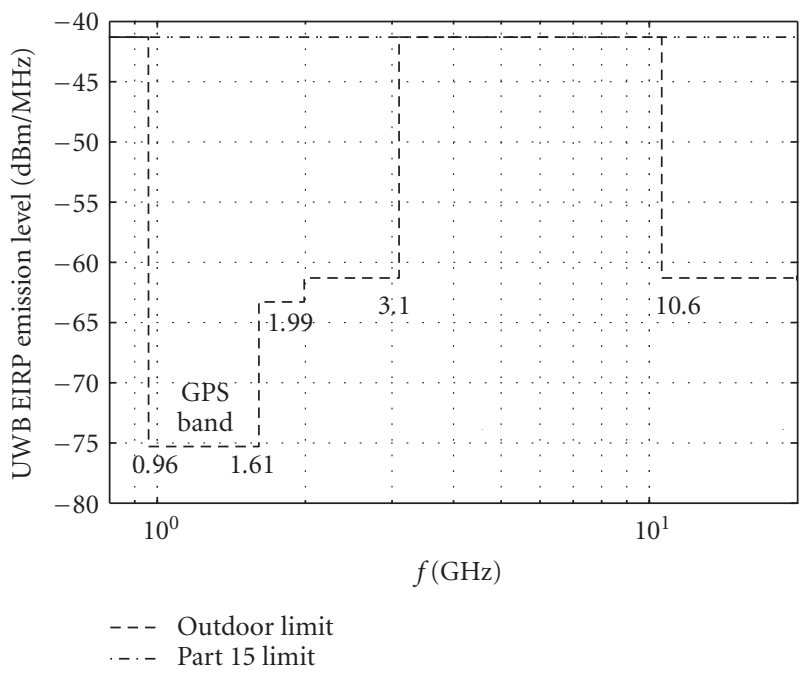

FIgURE 1: FCC ultra-wideband outdoor emission limits, bandwidth: $3.1 \mathrm{GHz}-10.6 \mathrm{GHz}, \quad-41.25 \mathrm{dBm} / \mathrm{MHz}$ EIRP (equivalent isotropic radiated power).

The drawbacks of the presently used coil, for inductive transmitting to the receiver (stimulator), are the high power consumption, the size, and optical reasons. On the other side the RF link is also used for power supply and no implanted battery is necessary. The big challenge is to substitute the inductive communication system with an alternative system like an infrared or a microwave link. A wireless ultrawideband (UWB) system is a new low power technology for reusing previously allocated RF bands without any licenses and by hiding the signals below the noise floor [2].

UWB signals are signals where the fractional bandwidth $\eta$ is greater than 0.2 or the signal occupies $0.5 \mathrm{GHz}$ or more of the spectrum [3]. The fractional bandwidth is defined as

$$
\eta=2 \frac{f_{H}-f_{L}}{f_{H}+f_{L}}
$$

where $f_{H}$ and $f_{L}$ are the frequencies measured at the $-10 \mathrm{~dB}$ emissions points. Operating over a large bandwidth, UWB or impulse radio can be classified as a spread spectrum technique for transmitting information. For high data rate applications (up to $500 \mathrm{Mbps}$ ) a DS-CDMA (direct-sequence code-division multiple access) and a multiband OFDM (orthogonal frequency-division multiplexing) system have been proposed to the IEEE 802.15.3a group for standardization [4]. On the other hand there is impulse radio (IR) which is carrierless and relies on a specific unchanging pulse-type waveforms to be transmitted in time with pulse-position modulation or on-off keying (OOK). IR transmitters and receivers are very elementary and of low power compared to DS-CDMA and OFDM. These single-period monopulses are very short in time duration and have a very low power spectral density across an ultrabroad frequency spectrum. The UWB transmitter and its antenna can be included into the speech processors case, the receiver and its antenna are beneath the skin and connected to the implant. The drawback using an UWB link, the lifetime of the implant's battery, is of capital importance, because for every changing, a surgery is necessary. The optimization of the implants power consumption demands highest priority.

\section{SPECIFICATIONS}

Nearly all parts of the transmitter and the receiver have to be implemented in a submicron CMOS process to minimize power and size. The power consumption for the receiver should be below $100 \mu \mathrm{W}$ as a mean value over time. The spectrum of the UWB signal has to fulfill the FCC requirements, shown in Figure 1, in order to allow coexistence with other systems $[3,5]$. The required transmission distances are 5 to $50 \mathrm{~mm}$ transcutaneous and $500 \mathrm{~mm}$ via air.

There should be a transcutaneous wireless connection between speech processor and implant and on the other hand optional features, like connections to a digital music player, a mobile phone, or another electronic audio device. It can also be envisaged to make stereo hearing possible by connecting the ipsilateral and the contralateral implants via the speech processor by an UWB wireless link, through or around the head. The antennas should be as small as possible and the radiated pulse should be able to penetrate human tissue with low attenuation. The safety issues for the patient concerning electromagnetic fields (EMF) are far below the restrictions compared with the EMF restrictions of the WHO (World Health Organization). In the United States for time-varying electric and magnetic fields from 0.3 to $6 \mathrm{GHz}$, a whole-body average specific absorption rate (SAR) of $0.08 \mathrm{~W} / \mathrm{kg}$ and a spatial peak SAR in the head of $1.6 \mathrm{~W} / \mathrm{kg}$ is permitted. If all the maximum output of an UWB device of $0.56 \mathrm{~mW}$ is absorbed by $1 \mathrm{~kg}$ of human tissue around the ear, it is still 2800 times below the critical spatial peak value. If we consider the free space loss of the link, then the absorbed power is again reduced by more than two decades. The input signal of our transmitter prototype is $\Sigma \Delta$-modulated with $1.2 \mathrm{Mbps}$, as it is also used by some CI manufacturers.

\section{TRANSMITTER}

The $1.2 \mathrm{Mbps} \Sigma \Delta$-modulated signal perfectly fits to on-off keying (OOK) modulation. OOK uses monophase modulation, where a pulse $w(t)$ transmits a "1" and no pulse transmits a "0" as shown in Figure 2 [6]. The transmitted signal can be defined as in (2). The symbol time $T_{S}$ is divided by the number of frames $N_{S}$ (number of pulses per symbol), whereas each frame lasts $T_{f}=T_{S} / N_{S}$ seconds. The discrete amplitude $a_{k}$ is " 1 " for a high bit $k$ and " 0 " for a low bit $k$.

For the system analysis it is convenient to consider a normalized energy for the pulse. Thus we will assume that the waveform used to transmit a bit has an energy $E_{b}$. This implies that the energy of a monocycle is $1 / N_{S}$ :

$$
s(t)=2 \sqrt{E_{b}} \sum_{k} a_{k} \sum_{n=0}^{N_{s}-1} w\left(t-n T_{f}-k T_{s}\right) .
$$




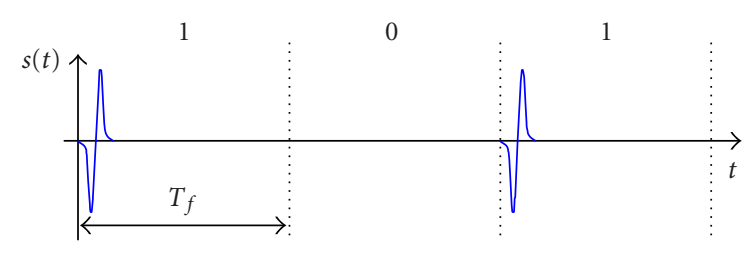

Figure 2: On-off keying principle.

One major problem in an UWB transmitter is the pulse generation with a pulse spectrum containing relevant power only above $3.1 \mathrm{GHz}$. The pulse duration for Gaussian monocycle pulses has to be below 300 ps for the spectral requirements; therefore CMOS or SiGe BiCMOS processes are required for fast pulse generation. Gerrits [7], Kim [8], and Azakkour [9] presented fundamentals to generate different pulse shapes on integrated circuits. However, CMOS is still too slow for $250 \mathrm{ps}$ pulses with an amplitude of $0.5 \mathrm{~V}_{\mathrm{pp}}$, but this goal will be reachable soon, due to rapid advances in semiconductor technology making an ultralow-power UWB transmitter feasible. For our prototype we used an ACT-TTL logic and a step recovery diode (SRD) pulse sharpener circuit. In Figure 3 the transmitter principle is shown [10]. The $50 \mathrm{MHz}$ pulse repetition frequency $(\mathrm{PRF})$ is $\sim 42$ times the symbol rate of $1.2 \mathrm{Mbps}$. This allows to transmit several pulses per bit which guarantees a higher interference resistance and a lower power spectral density. The transmitter output signal is shown in Figure 4. The problem with this prototype (see Figure 5) is the high bias current of the SRD; hence for a low power application a transmitter in CMOS technology is obligatory. By using fast SiGe bipolar transistors (BFP 640F, etc.) instead of the SRD, the average transmitter power can be dropped below $1 \mathrm{~mW}$ and the pulses become two times faster than with a SRD pulser. Further research work is planned in this field.

\section{ANTENNA}

Ultra-wideband antennas are nowadays of huge interest based on the enormous demand in broadband wireless systems. Because of the small power levels authorized by the FCC, every tenth of a dB counts in an UWB system [11]. For our prototype we used very simple monopole antennas and planar dipole antennas shown in Figure 6 and described in [6].

The antenna, a $\lambda / 2$ monopole or Marconi antenna, was designed for a center frequency of $5 \mathrm{GHz}$. The ground plane is the outer conductor of the SMA connector. The VSWR (voltage standing wave ratio) of the manually enfolded monopole antenna exhibits in the range of $1.8 \mathrm{GHZ}$ to $3.7 \mathrm{GHz}$ a wanted poor matching, which results in nearly no radiation below $3.7 \mathrm{GHz}$; therefore no filter to fulfill the FCC requirements is needed. The planar dipole antenna has a much better filter performance at an attenuation difference of $20 \mathrm{~dB}$ between 2 and $4 \mathrm{GHz}$ as shown in Figure 7a. For our prototype the monopole antenna was used, because the output pulses of the transmitter are not short enough to send the whole power above $3 \mathrm{GHz}$. The spectrum of the transmitted signal produced by the transistor pulser and shaped by the dipole antenna is shown in Figure 7b. Presently the spectrum of the transmitted signal is not FCC compliant. A better fit into the FCC spectrum will be reached by using a bipolar transistor pulse generator. This future pulse signal will result in a higher attenuation through the human tissue, but a FDTD (finite difference time domain) antenna simulation can improve the matching, the gain, and the filter characteristics and will compensate this problem.

\section{RECEIVER}

An asynchronous or noncoherent receiver is sufficient for a communication link from the speech processor to the implant. The first proposed receiver prototype [6] consisted of a low noise amplifier (LNA), a detector diode, or a detector IC, which rectifies the signal and a lowpass filter for recovering the envelope and an operational amplifier which amplifies the signal back to a TTL level [12]. This type of receiver is not applicable for implantation into the head, because of its high power consumption in the $10-100 \mathrm{~mW}$ range. For less power consumption tunnel diodes or back tunnel diodes can be used. In the tunnel diode, the semiconductor materials used for forming a junction are doped to the extent of one thousand impurity atoms per ten million semiconductor atoms. This heavy doping produces an extremely narrow depletion zone similar to that in a Zener diode. Also because of the heavy doping, a tunnel diode exhibits an unusual currentvoltage characteristic curve as compared with that of an ordinary junction diode. The characteristic of a tunnel diode is illustrated in Figure 8. The three most important aspects of this characteristic are the forward current increases to a peak $\left(I_{P}\right)$ with a small applied forward bias, the decreasing forward current with an increasing forward bias to a minimum valley current $\left(I_{V}\right)$, and the increasing forward current with a further increase in the bias voltage. The part of the characteristic curve between $I_{P}$ and $I_{V}$ is the region of negative resistance. Backward diodes or back tunnel diodes (BTD) are tunnel diodes with a maximum forward voltage of $\sim 100 \mathrm{mV}$ and a minimum reverse voltage of $\sim 400 \mathrm{mV}$. These diodes operate in reverse mode as a very sensitive pulse detector $(\sim 1000 \mathrm{mV} / \mathrm{mW})$. Because of the high sensitivity no more LNA is needed. The drawbacks of diode biasing and LNA power consumption disappear when using this diode. By using back tunnel diodes it should be possible to fulfill the $<100 \mu \mathrm{W}$ power consumption requirement for the receiver.

The backward diode is connected in reverse direction and rectifies very small signals down to $-15 \mathrm{dBm}$ without any biasing as a real passive part. The prototyped receiver only consists of a BTD and a resistor connected to a $2 \mathrm{MHz}$ lowpass filter. The rectified output signal of the receiver is plotted in Figure 9a. The envelope of this signal recovers the $1.2 \mathrm{Mhz}$ input signal as shown in Figure 9b. 

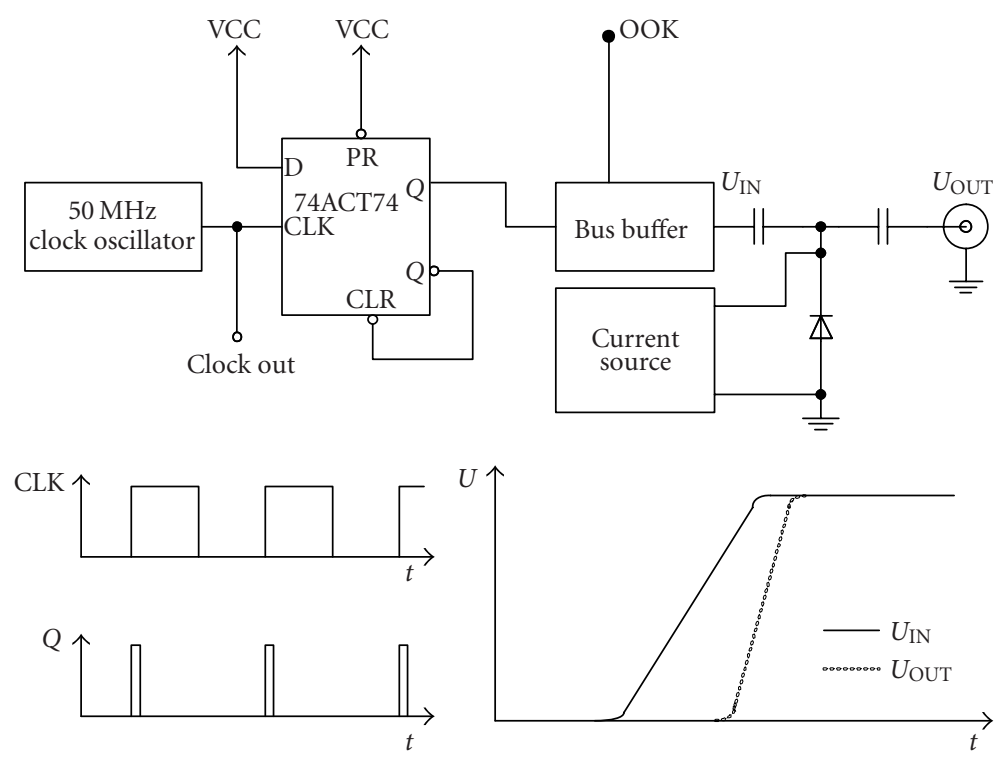

FIGURE 3: UWB transmitter with OOK and SRD pulse sharpener.

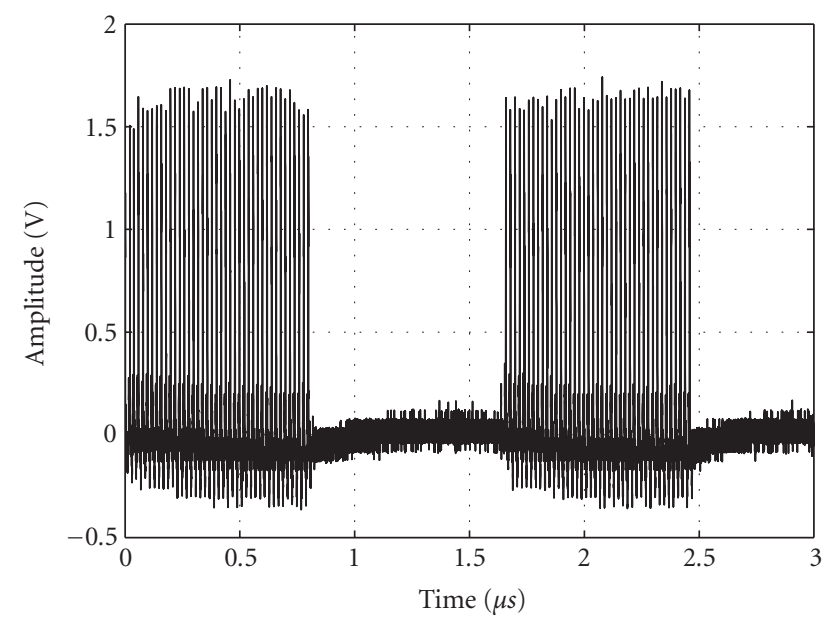

FIGURE 4: OOK transmitter output, PRF $=50 \mathrm{MHz}$, symbol rate is 1.2 Mbps.

\section{RESULTS}

The SRD transmitter shown in Figure 5 and three different detector receivers with an LTC5508 (7 GHz RF power detector from Linear Technologies), a tunnel diode receiver, and a back tunnel diode receiver have been used for prototype UWB systems. If a hand encloses each antenna, as shown in Figure 10, the signal is still received perfectly. In Table 1 the three different receiver prototypes are compared in relation to input power range, sensitivity, and power consumption.

The best results in terms of power consumption are obtained with a back tunnel diode. The power consumption of the receiver can be minimized to zero, because it is a passive detector, but there is the drawback of a shorter propagation distance by the minimum input power of $-15 \mathrm{dBm}$. The only power needed is for an active lowpass filter after

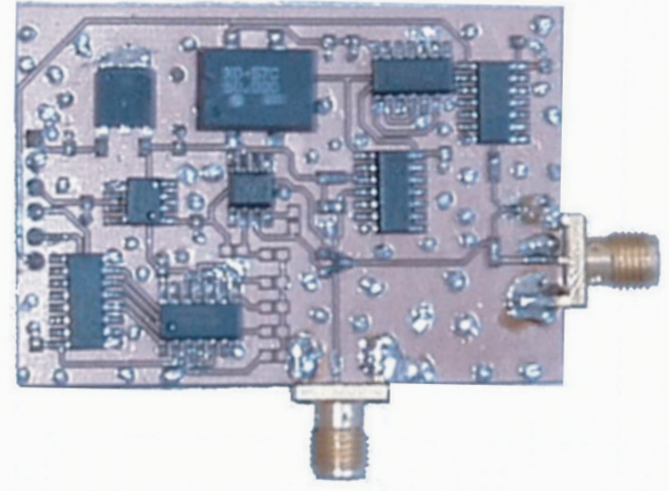

FIGURE 5: UWB transmitter prototype.

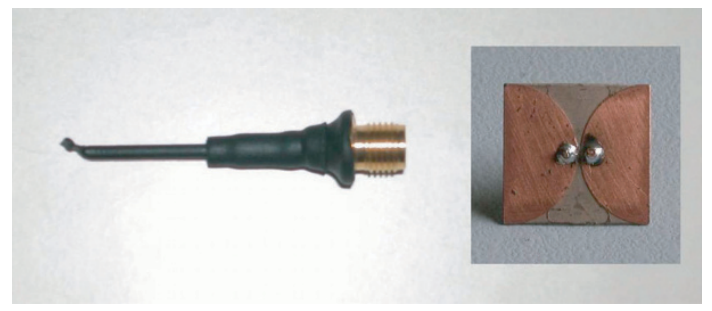

Figure 6: $30 \mathrm{~mm}$ monopole antenna (left) and a $20 \times 20 \mathrm{~mm}^{2}$ planar dipole antenna (right).

the receiver, which can be easily implemented with less than $100 \mu \mathrm{W}$ consumption. The output of the lowpass filter is fed to the stimulator. If a person has two CIs, with a transceiver in each ear, it should be possible to connect the two CIs over 


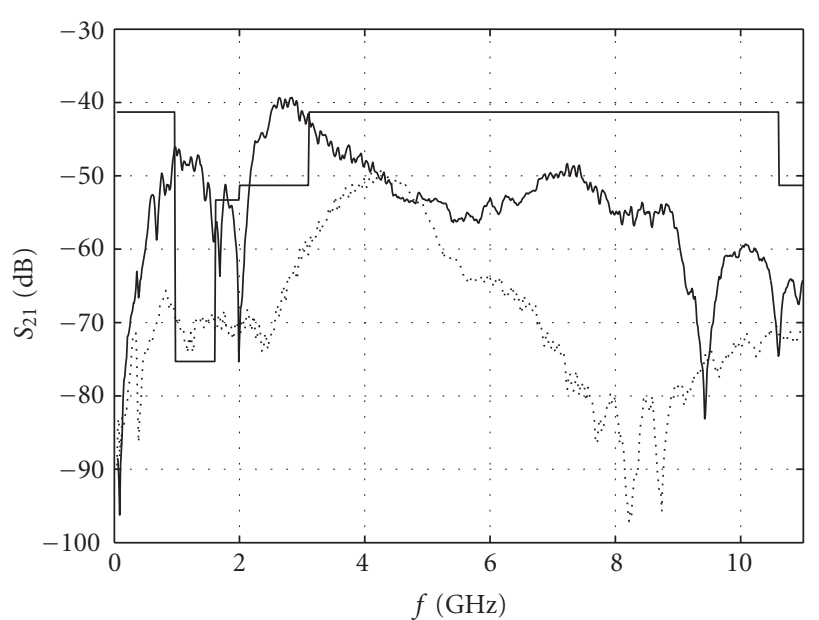

(a)

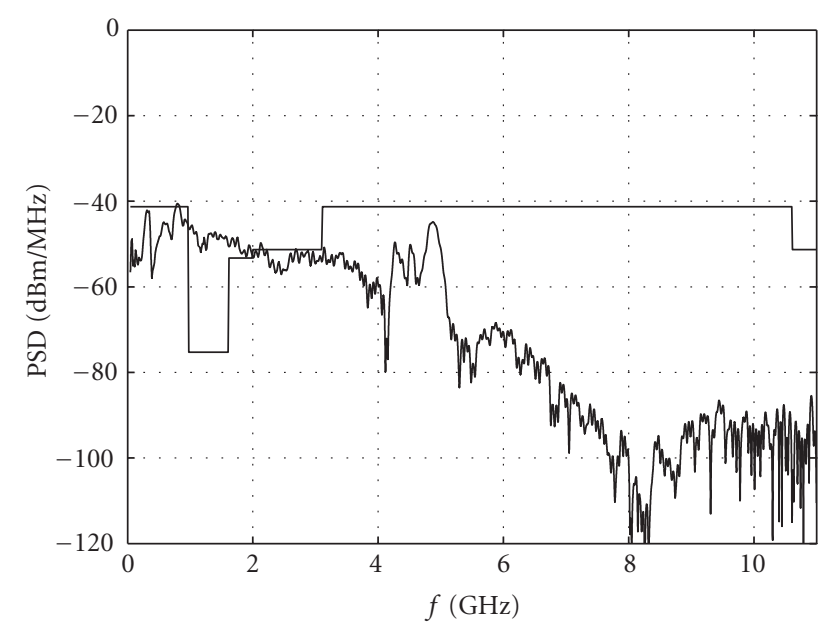

(b)

FIgURE 7: (a) $S_{21}$ of a planar dipole antenna pair (dotted line) and a monopole antenna pair (solid) over $50 \mathrm{~mm}$ line of sight distance together with the FCC spectrum mask. (b) Transmitted signal and FCC indoor spectrum mask.

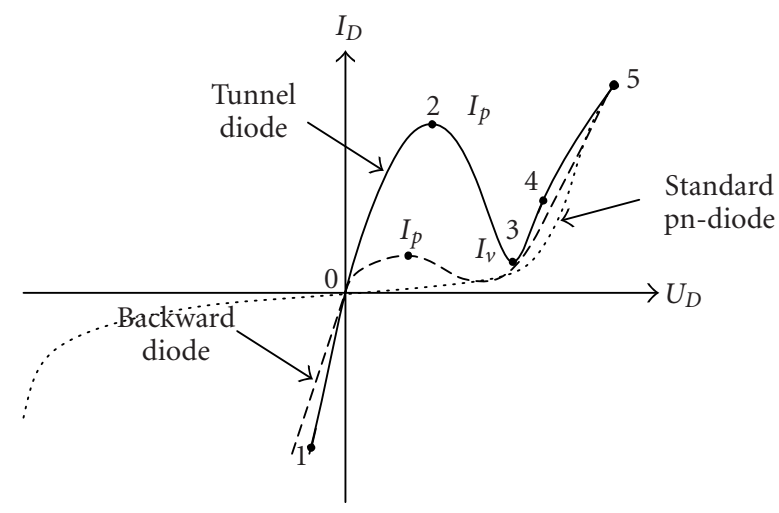

Figure 8: Characteristic diagram of a tunnel diode.

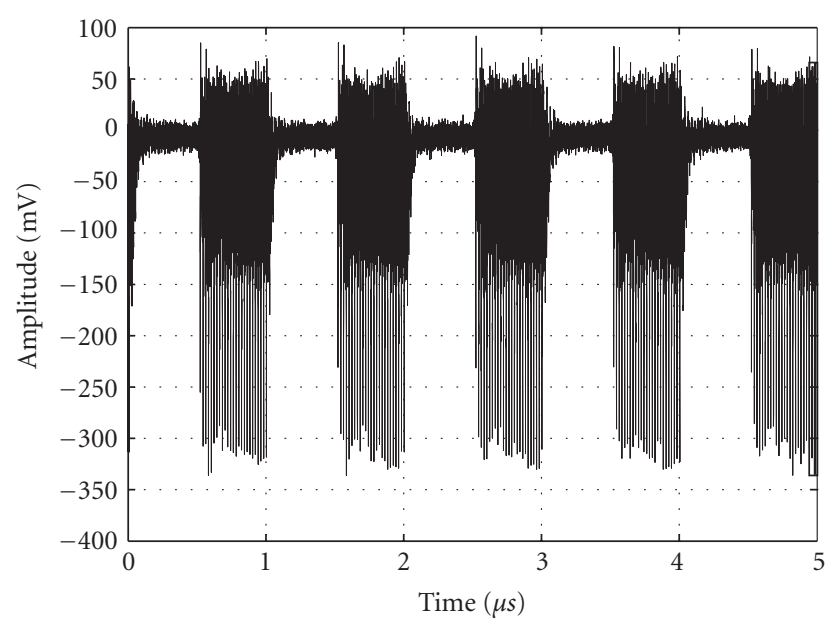

(a)

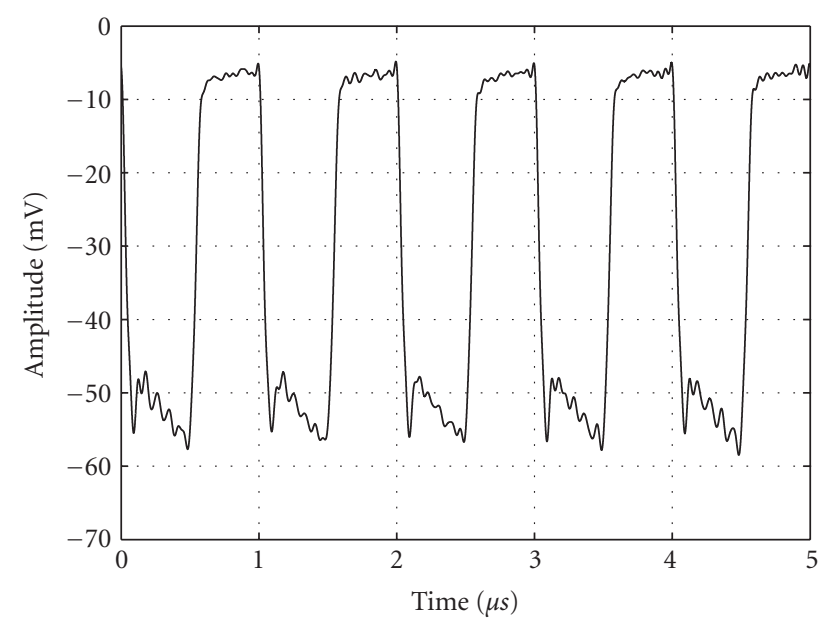

(b)

FIGURE 9: (a) Output of the BTD receiver and (b) output of the $2 \mathrm{MHz}$ lowpass filter.

a wireless UWB link with the speech processor. This offers new solutions in stereo hearing for deaf persons and generates a big challenge for the implementation of the required digital signal processing. Interference between the transmitters can be eliminated with a transmit protocol controlled by the speech processor. A back-telemetry link with a low power transistor pulser in the implant is of high importance for the transmit protocol. If it is at a very low rate the battery lifetime in the implant will not be stressed too hard. The quality of the communication link is dependent on the distance. The LTC 5508 receiver had a good transmission quality for distances up to $500 \mathrm{~mm}$, with the back tunnel diode the quality gets week at distances longer than $80 \mathrm{~mm}$, in transcutaneous (through two hands) propagation channels. 


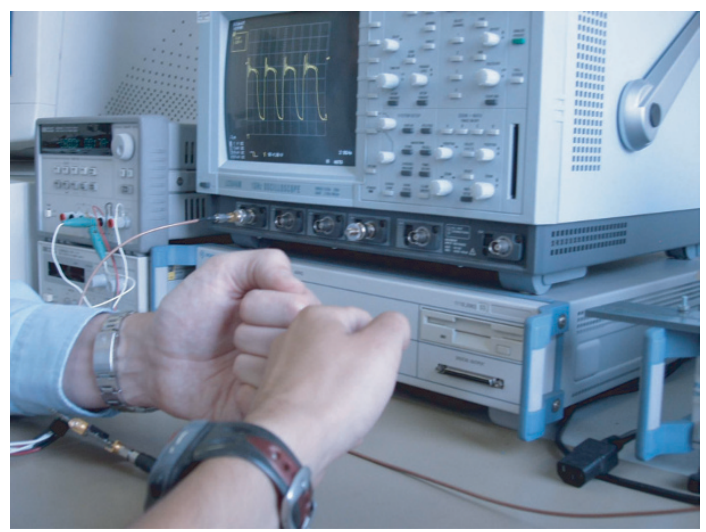

FIgURE 10: Transmitting through human tissue.

TABLE 1: Comparison of different detector receivers.

\begin{tabular}{lcc}
\hline $\begin{array}{l}\text { Type of detector } \\
\text { receiver }\end{array}$ & $\begin{array}{c}\text { Input power range } \\
\text { (sensitivity) }\end{array}$ & Power consumption \\
\hline $\begin{array}{l}\text { Receiver with } \\
\text { LTC5508 + LNA }\end{array}$ & $-30 \mathrm{dBm}-12 \mathrm{dBm}$ & $50 \mathrm{~mW}$ \\
\hline $\begin{array}{l}\text { Tunnel diode } \\
\text { receiver }\end{array}$ & $\begin{array}{c}-38 \mathrm{dBm}-5 \mathrm{dBm} \\
(600 \mathrm{mV} / \mathrm{mW})\end{array}$ & $\begin{array}{c}2-10 \mathrm{~mW}, \\
\text { biasing necessary }\end{array}$ \\
\hline $\begin{array}{l}\text { Back tunnel } \\
\text { diode receiver }\end{array}$ & $\begin{array}{c}-15 \mathrm{dBm}-5 \mathrm{dBm} \\
(800 \mathrm{mV} / \mathrm{mW})\end{array}$ & $\begin{array}{c}0, \\
\text { no biasing }\end{array}$ \\
\hline
\end{tabular}

\section{CONCLUSION}

An electronic hearing apparatus, like the cochlear implant with its low power consumption requirements, is an ideal application for an UWB communication link. A prototype system for this application has been proposed. The OOK transmitter with a data rate of $1.2 \mathrm{Mbps}$ has a pulse repetition frequency of $50 \mathrm{MHz}$ and is based on pulse generation with step recovery diodes. The monopole antenna and the planar dipole antenna have a good performance in radiating through human tissue. Three different detector receivers with a ready detector IC, tunnel diodes, and backward diodes have been compared in relation to power consumption and sensitivity. The use of UWB communication systems for cochlear implants will become soon realistic if all the components can be integrated in a fast CMOS process and the pulse detection in the receiver can be performed by a passive element like the back tunnel diode, to reach a long battery lifetime of the implant. Because of the low detection sensitivity of back tunnel diodes the propagation distance can only be increased by a higher antenna gain, which is generally dependent on the directivity and the size. Improvement reserves for the proposed prototypes are the use of faster and more broadband pulses, antennas with a better performance, and more sensible detector receivers.

\section{ACKNOWLEDGMENTS}

This work was carried out at the Institute for Communication and Information Engineering (ICIE), University of Linz, and was funded by the Linz Center of Competence in Mechatronics (LCM). We would like to acknowledge Ralf Ruders- dorfer and Gerhard Kaineder for their help in manufacturing the test hardware.

\section{REFERENCES}

[1] P. C. Loizou, "Mimicking the human ear," IEEE Signal Processing Mag., vol. 15, no. 5, pp. 101-130, 1998.

[2] K. Siwiak, "Ultra-wide band radio: introducing a new technology," in Proc. IEEE VTS 53rd Vehicular Technology Conference (VTC '01), vol. 2, pp. 1088-1093, Rhodes, Greece, May 2001.

[3] First Report and Order, in "FCC; Washington, DC, USA," 2002, FCC, Washington, DC, USA, p. 118.

[4] http://www.ieee802.org/15/pub/TG3a.html, IEEE 802.15 WPAN High Rate Alternative PHY Task Group 3a (TG3a).

[5] UWB Emmission Limits, 2002, FCC, Washington, DC, USA, p. 4.

[6] T. Buchegger, G. Oßberger, E. Hochmair, U. Folger, A. Reisenzahn, and A. Springer, "An ultra low power transcutaneous impulse radio link for cochlea implants," in Proc. International Workshop on Ultra Wideband Systems (IWUWBS '04) \& Conference on Ultra Wideband Systems and Technologies (UWBST '04), Kyoto, Japan, May 2004.

[7] J. F. M. Gerrits and J. R. Farserotu, "Wavelet generation circuit for UWB impulse radio applications," IEE Electronics Letters, vol. 38, no. 25, pp. 1737-1738, 2002.

[8] H. Kim, D. Park, and Y. Joo, "Design of CMOS Scholtz's monocycle pulse generator," in Proc. IEEE Conference on Ultra Wideband Systems and Technologies (UWBST'03), pp. 81-85, Reston, Va, USA, November 2003.

[9] A. Azakkour, M. Regis, F. Pourchet, and G. Alquie, "Challenges for a new integrated ultra-wideband (UWB) source," in Proc. IEEE Conference on Ultra Wideband Systems and Technologies (UWBST'03), pp. 433-437, Reston, Va, USA, November 2003.

[10] T. Buchegger, G. Oßberger, A. Reisenzahn, A. Stelzer, and A. Springer, "Pulse delay techniques for PPM impulse radio transmitters," in Proc. IEEE Conference on Ultra Wideband Systems and Technologies (UWBST '03), pp. 37-41, Reston, Va, USA, November 2003.

[11] H. G. Schantz, "Introduction to ultra-wideband antennas," in Proc. IEEE Conference on Ultra Wideband Systems and Technologies (UWBST '03), pp. 1-9, Reston, Va, USA, November 2003.

[12] A. Reisenzahn, "Hardwarekomponenten für Ultra-Wideband Radio," Diploma thesis, Institute for Communication and Information Engineering, Johannes Kepler Universität, Linz, Austria, 2003.

Thomas Buchegger was born in Linz, Austria, in 1974. He received the Dipl.-Ing. degree in mechatronical engineering from the Johannes Kepler University, Linz, Austria, in 2002 and is now working on his Dr. techn. (Ph.D.) degree at the Institute for Communications and Information Engineering. $\mathrm{He}$ is employed at Linz Center of Competence in Mechatronics for the strategic project "Wireless Sensing, Data and Communication for Machinery and Vehicles." His areas of experience are ultra-wideband sub-nanosecond pulse generation, system design for ultra-wideband communication systems, and transmitted reference pulse communication systems.

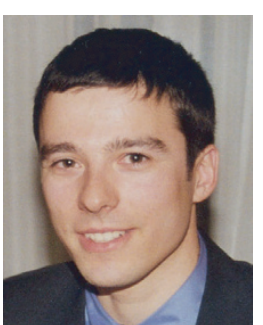


Gerald Oßberger was born in Linz, Austria, in 1975. He received the Digl.-Ing. (M.S.) degree in mechatronical engineering from Johannes Kepler University of Linz, Austria, in 2002. He is currently pursuing the Ph.D. degree in mechatronical engineering at the Johannes Kepler University of Linz, Austria. In 2002 he joined the Linz Center of Competence in Mechatronics $\mathrm{GmbH}$ as a Research Assistant. His primary research inter-

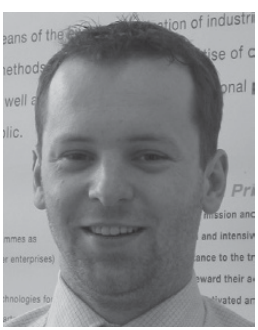
ests are in electromagnetics, pulse-based UWB ground penetrating, and signal processing for detection and classification of buried landmines.

Alexander Reisenzahn was born in Wels, Austria, in 1973. He received the Dipl.-Ing. degree in mechatronics from the Johannes Kepler University, Linz, Austria, in 2004. Since 2004, he has been with the Institute for Microelectronics at the Johannes Kepler University, Linz, Austria, and is currently working toward the Dr. techn. (Ph.D.) degree. His focus is on the design of hardware for ultra-wideband applications.

Erwin Hochmair received the Dipl.-Ing. and Dr. techn. degrees in electrical engineering from the Technical University of Vienna, Austria, in 1964 and 1967, respectively. Since 1986 he is a Full Professor of applied physics at the University of Innsbruck, Austria. He held positions at the Technical University of Vienna, Austria, the NASA Marshall Space Flight Center in Huntsville, Ala, and at the Stanford University, Stan-

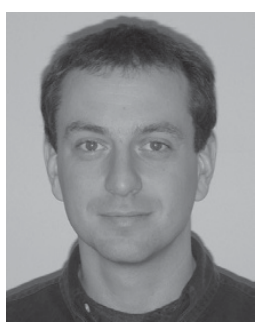
ford, Calif. From 1993 to 1995 he was Dean of the Faculty of Natural Sciences at the University of Innsbruck. His research interests encompass circuit design, RF engineering, and signal processing applications, and focus on many aspects of cochlear implant design. He has (co-)authored over 100 technical articles and holds about 50 patents. He is the recipient of the 1977 Best Paper Award at the IEEE International Solid State Circuits Conference, the HolzerPreis of the TU Vienna, the Erwin Schrödinger Preis of the Austrian Academy of Sciences 2003, and has been awarded an honorary Dr. med. degree from the Technical University of Munich in 2004. Together with his wife, who also holds several degrees in electrical engineering, he has founded several companies in the field of biomedical engineering.

Andreas Stelzer was born in Haslach an der Mühl, Austria, in 1968. He received the Dipl.-Ing. degree in electrical engineering from the Technical University of Vienna, Austria. In 2000 he received the Dr. techn. degree (Ph.D.) in mechatronics with honors "sub auspiciis praesidentis rei publicae" from the Johannes Kepler University. In 2003 he became an Associate Professor at the same university. Since 2000 he has been with the Institute for Communications and Information Engineering. At the European Microwave Week 2003 he received an EEEfCOM Innovation Award and he was corecipient of the Best Paper Award at the European Radar Conference. Since 2003 he has served
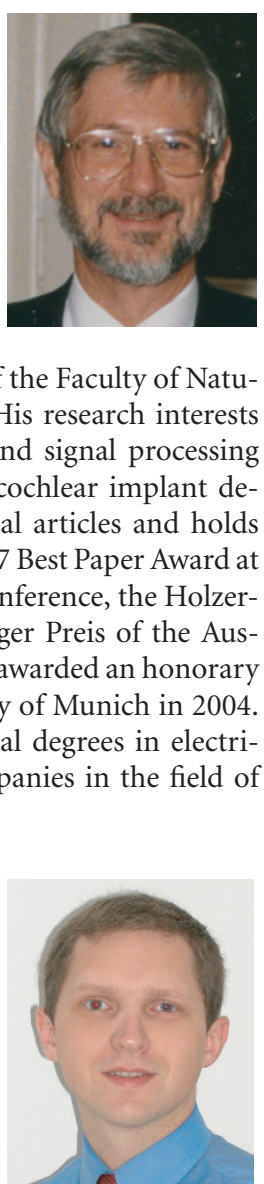

as a "Key Researcher" for the Linz Center of Competence in Mechatronics and is responsible for numerous projects with industrial partners. He has authored or coauthored more than 100 journal and conference papers. His research work focuses on microwave sensor systems for industrial applications, RF and microwave subsystems, ultra-wideband technology, SAW sensor systems and applications, as well as digital signal processing for sensor signals. Dr. Stelzer is a Member of the Austrian OVE and IEEE. He served as an Associate Editor for the IEEE Microwave and Wireless Components Letters.

Andreas Springer received the Dipl.-Ing. degree in electrical engineering from the Technical University of Vienna, Austria, in 1991, and the Dr. techn. (Ph.D.) degree and the Univ.-Doz. (Habilitation) degree both from the University of Linz, Austria, in 1996 and 2001, respectively. From 1991 to 1996 he was with the Microelectronics Institute at the University of Linz, Austria. In 1997, he joined the Institute for Communications

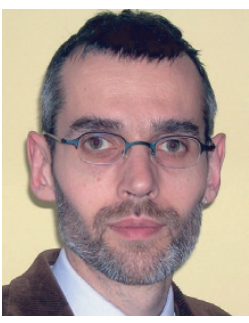
and Information Engineering at the same university, where he became a Full Professor in 2005. Since July 2002 he has been also Head of the Institute for Communications and Information Engineering at the University of Linz. He has been engaged in research work on GaAs integrated millimeter-wave TEDs, MMICs, and millimeter-wave sensor systems. His current research interests are focused on simulation of wireless communication systems, spread spectrum communications, single-carrier and multicarrier communications, UWB, direct conversion architectures, equalization, MIMO, UMTS, and RFICs. In these fields, he has published more than 100 papers in journals and at international conferences. 